\title{
A THz Coupler based on Graphene Patterns with SU- 8 Photoresist Dielectric Layer
}

Elham Zandi ( $\sim$ Elham.Zandi2021@gmail.com )

Islamic Azad University Saveh Branch https://orcid.org/0000-0002-9977-1459

\section{Research Article}

Keywords: THz, Multi-Layer, Graphene, Equivalent Circuit Model, Coupler, SU-8 photoresist dielectric layer

Posted Date: November 17th, 2021

DOI: https://doi.org/10.21203/rs.3.rs-1057240/v1

License: (9) This work is licensed under a Creative Commons Attribution 4.0 International License. Read Full License 


\title{
A THz Coupler based on Graphene Patterns with SU-8 Photoresist Dielectric Layer
}

\author{
Elham Zandi
}

Department of Electrical and Electronic Engineering, Islamic Azad University, Saveh Branch, Saveh, Iran.

\begin{abstract}
:
Leveraging both method and concept, a novel multi-layer structure based on Graphene patterns and SU-8 photoresist dielectric is proposed at $\mathrm{THz}$ frequency range. By considering reflection and transmission channels as outputs, a simple THz coupler is provided. The structure is described exploiting equivalent circuit model while results are verified by full wave simulations. According to simulation results, the proposed device is able to reflect and transmit THz waves with high sensitivity versus gate biasing. The operation involves five bands in entire THz spectrum while the structure behavior can be adjusted by external gate biasing. Such a tunable device is in great demand to realize optical sensors and systems in several fields from indoor communications, security and medical imaging.

Keywords: THz, Multi-Layer, Graphene, Equivalent Circuit Model, Coupler, SU-8 photoresist dielectric layer.
\end{abstract}

\section{Introduction:}

The terahertz (THz) band is the frequency band between the upper limit of electronics and the lower limit of photonics. This band has been intensively considered by researchers from several fields due to speedier operations compared to RF electronics while reduces the risks of high frequency in the photonics [1-3]. For instance, medical imaging can be performed via $\mathrm{THz}$ radiation to reduce hazardous effects on body organs. Now, to realize applications in the THz spectrum, we need materials with appropriate features. Graphene is a suitable option for implementing THz devices [4]. In 2010, the Nobel Prize in Physics was awarded to successful researches on graphene. These studies have identified graphene as a two- 
dimensional carbon material, as thick as a single layer of carbon atoms $(0.32 \mathrm{~nm})$ and in a honeycomb network [5]. The density of graphene carriers can be adjusted by changing the gate voltage applied to the graphene. The density of carriers can be affected by electrical doping (via gate voltage) and chemical doping. In addition, it has been observed that the experimental results of high-density graphene carriers $\left(n>10^{12} \mathrm{~cm}^{-2}\right)$ can well be described as a system with one type of carrier, type $\mathrm{n}$. The electrons in graphene behave like Dirac fermions without mass. Their distribution on the energy level in a system can be described by the Fermi-Dirac distribution function (the Fermi function) as shown by Eq. (1) [6].

$$
F_{D}(E)=\frac{1}{e^{\frac{E-\mu_{c}}{K_{B} T}}+1}
$$

Where:

\begin{tabular}{|l|l|}
\hline$E$ & Energy level. \\
\hline$\mu_{C}=e E_{F}$ & Chemical potential and dependent of temperature. \\
\hline$K_{B}$ & Boltzmann constant. \\
\hline$T$ & Temperature. \\
\hline
\end{tabular}

From view point of energy levels, the density of electrons (n) in graphene can be shown by Eq. (2).

$$
n=\frac{2}{\pi \mathrm{h}^{2} v_{F}^{2}} \int_{0}^{\infty} E\left[F_{D}(E)-F_{D}\left(E+2 \mu_{c}\right)\right] d E
$$

Where:

\begin{tabular}{|l|l|}
\hline$v_{F}$ & Fermi velocity: $v_{F}=1.0 \times 10^{6} \frac{\mathrm{m}}{\mathrm{s}}$. \\
\hline $\mathrm{h}$ & Reduce Plank constant. \\
\hline$F_{D}$ & Fermi-Dirac distribution function. Report in Eq. (1).
\end{tabular}

If the chemical potential is greater than the thermal energy ( $\mu_{c} \gg K_{B} T$ ) Eq. (2) can be simplified Eq. (3).

$$
n=\frac{2}{\pi \mathrm{h}^{2} v_{F}^{2}}
$$


When the gate voltage is applied to graphene, the density of electrons induced by the gate voltage can be described as Eq. (4).

$$
n=C_{\text {gate }} \times \frac{V_{G}-V_{C N P}}{e}
$$

Where:

\begin{tabular}{|l|l|}
\hline$C_{\text {gate }}=\frac{\varepsilon_{\text {insulator }}}{t_{\text {insulator }}}$ & Gate capacity per unit area. \\
\hline$\varepsilon_{\text {insulator }}$ & Insulation permeability. \\
\hline$t_{\text {insulator }}$ & Insulation thickness under graphene. \\
\hline$V_{G}$ & Gate voltage applied. \\
\hline$V_{C N P}$ & Charge Neutral Point Voltage \\
\hline$e$ & Electron charge: $e=1.602 \times 10^{-19} \mathrm{C}$ \\
\hline
\end{tabular}

Another feature of electrons in graphene is the mobility of graphene electrons $(\mu)$. This parameter determines how fast the electron can move in the graphene plate. Suspended graphene (graphene that is free on both sides) has been reported to have equal electron mobility $\left(\mu=200,000 \frac{\mathrm{cm}^{2}}{V s}\right)$ for the density of carriers at low temperatures ( $\left.n=5 \times 10^{11} \mathrm{~cm}^{-2}\right)$. However, at room temperature $\left(T=300^{\circ} \mathrm{K}\right)$, increasing the resistance reduces the mobility $\left(\mu=120,000 \frac{\mathrm{cm}^{2}}{\mathrm{Vs}}\right)$. Increased strength can also come from the ribbonshaped graphene edge, and the metal connections of the gate-voltage structures. By considering the resistance and voltage of the gate, the mobility of graphene can be calculated. Via Eq. (5) [7].

$$
\mu=\frac{1}{n e \rho}
$$

Where:

\begin{tabular}{l|l}
$n$ & Electrons density of induced in the gate and defined in Eq. (4). \\
$\rho$ & Electrical resistance of graphene.
\end{tabular}


In addition, substrates also affect the mobility of electrons in graphene. For graphene sheets on the substrate $\mathrm{SiO}_{2}$, the electron scattering mechanism further reduces the mobility of the electrons $\mu=40,000 \frac{\mathrm{cm}^{2}}{V s}$. Compared to the substrate $\mathrm{SiO}_{2}$, the mobility of electrons in graphene on the hexagonal boron nitride substrate $h-B N$ is higher. From the view point of scattering mechanism, the mobility of electrons can be described as Eq. (6):

$$
\mu=\frac{L e v_{F}}{\mu_{c}}=\frac{e \tau v_{F}^{2}}{\mu_{c}}=\frac{e v_{F}^{2}}{\Gamma \mu_{c}}
$$

where

$$
\begin{array}{l|l}
L=\tau v_{F} & \text { Free path distance of electrons. } \\
\hline \tau=\frac{1}{\Gamma} & \text { Electron relaxation time. } \\
\hline \Gamma & \text { Electron scattering rate } \\
\hline
\end{array}
$$

The relaxation time of electrons $(\tau)$ is another parameter that is very important in the behavior of electrons in graphene. This parameter describes the time between two events of scattering during the electron motion process. This plays a key role in research on graphene material and its conductivity. The conductivity of graphene has two components: intra-band transfer and inter-band transfer, which according to Kubo's formula is shown as Eq. (8) [8].

$$
\begin{aligned}
& \sigma(\omega)=\frac{j e^{2}(\omega-2 j \Gamma)}{\pi \mathrm{h}^{2}} \times \\
& {\left[\frac{1}{(\omega-2 j \Gamma)^{2}} \int_{0}^{\infty} \varepsilon\left(\frac{\partial f_{d}(\varepsilon)}{\partial \varepsilon}-\frac{\partial f_{d}(-\varepsilon)}{\partial \varepsilon}\right) d \varepsilon-\int_{0}^{\infty} \frac{f_{d}(-\varepsilon)-f_{d}(\varepsilon)}{(\omega-2 j \Gamma)^{2}-4\left(\frac{\varepsilon}{\mathrm{h}}\right)^{2}} d \varepsilon\right]}
\end{aligned}
$$

Here, the first part of Eq. (8) is known as the intra-band conduction ( $\sigma(\omega)_{\text {intra }}$ ), which is the result of the intra-band transfers of electrons. And the second part, which results from the inter-band transfers of electrons, is known as the inter-band conductivity $\left(\sigma(\omega)_{\text {inter }}\right)$. Intraband conduction is obtained from the Drude model and is described as Eq. (9) [10-12].

$$
\sigma(\omega)_{\text {intra }}=-\frac{j e^{2} K_{B} T}{\pi \mathrm{h}^{2}(\omega-2 j \Gamma)}\left(\frac{\mu_{c}}{K_{B} T}+2 \ln \left(\frac{1}{e^{\frac{\mu_{c}}{K_{B} T}}+1}\right)\right)
$$

If $\left|\mu_{c}\right|, \mathrm{h} \omega>>K_{B} T$, also, inter-band conductivity defined as Eq. (10). 


$$
\sigma(\omega)_{\text {inter }} \cong-\frac{j e^{2}}{4 \pi \mathrm{h}^{2}} \ln \left(\frac{2\left|\mu_{c}\right|-(\omega-2 j \Gamma) \mathrm{h}}{2\left|\mu_{c}\right|+(\omega-2 j \Gamma) \mathrm{h}}\right)
$$

Inter-band conductivity only when: h $\omega$ or $E_{E M}>>2 \mu_{c}$

Eq. (11) also shows graphene coefficient electrical permittivity of graphene:

$$
\varepsilon_{\text {Graphene }}=1+\mathrm{j} \frac{\sigma_{\text {Grhapene }}}{\omega \cdot \varepsilon_{0} \cdot \mathrm{t}_{\text {Grhaphene }}}
$$

Where:

\begin{tabular}{|l|l|}
\hline$\sigma$ & Graphene conductivity. \\
\hline$t$ & Graphene thickness. \\
\hline$\varepsilon_{0}=\frac{1}{\mu_{0} v_{0}^{2}}$ & Vacuum permittivity \\
\hline$\mu_{0}$ & Magnetic permeability \\
\hline$v_{0}$ & Light velocity in a vacuum \\
\hline
\end{tabular}

To find the relationship between the electrical permittivity and the conductivity of graphene, consider graphene as a very thin material at an angular frequency $(\omega)$. The complex electrical permittivity is defined as Eq. (12) [13-14].

$$
\begin{aligned}
& \varepsilon_{G}=1+j \frac{\sigma_{G}}{\omega \cdot \varepsilon_{0} \cdot t_{G}} \stackrel{\sigma_{g}=\sigma_{\mathrm{Re}}+j \sigma_{\mathrm{Im}}}{\longrightarrow} \\
& =1+j \frac{\operatorname{Re}\left(\sigma_{G}\right)+j \operatorname{Im}\left(\sigma_{G}\right)}{\omega \cdot \varepsilon_{0} \cdot t_{G}} \\
& =1+j \frac{\operatorname{Re}\left(\sigma_{G}\right)}{\omega \cdot \varepsilon_{0} \cdot t_{G}}-\frac{\operatorname{Im}\left(\sigma_{G}\right)}{\omega \cdot \varepsilon_{0} \cdot t_{G}} \\
& =\left(1-\frac{\operatorname{Im}\left(\sigma_{G}\right)}{\omega \cdot \varepsilon_{0} \cdot t_{G}}\right)+j \frac{\operatorname{Re}\left(\sigma_{G}\right)}{\omega \cdot \varepsilon_{0} \cdot t_{G}}
\end{aligned}
$$

In conclusion:

$$
\begin{aligned}
& \varepsilon_{G}=\varepsilon_{G_{-} \text {real }}+j \varepsilon_{G_{-} \text {imag }} \rightarrow \\
& \operatorname{Re}\left(\varepsilon_{G}\right) \propto 1-\frac{\operatorname{Im}\left(\sigma_{G}\right)}{\omega \cdot \varepsilon_{0} \cdot t_{G}} \\
& \operatorname{Im}\left(\varepsilon_{G}\right) \propto \frac{\operatorname{Re}\left(\sigma_{G}\right)}{\omega \cdot \varepsilon_{0} \cdot t_{G}}
\end{aligned}
$$


A significant issue in the above equation is the relationship between the imaginary part of the conductivity and the real part of the electrical permittivity. The real conductivity part is also related to the imaginary part of the graphene electrical permittivity. Since the losses in graphene are modeled with the imaginary part of its electrical permittivity, the above relation shows that the losses in graphene depend on the real part of its conductivity, and we can use the graphene adjustability to form the losses in the form of desirable change. Knowledge of graphene material and the behavior of electrons in graphene now makes it possible to implement graphene-based devices in the terahertz band. As a result, numerical modeling methods as well as circuit modeling methods paved the way for the design of such devices.

Here in this work, four layers of graphene patterns are attached to three layers of dielectrics to create two channels as output and coupled ports. Next section describes the proposed device and equivalent circuit model with details while section 3 reports simulation results of the proposed structure. Finally, conclusion is brought.

\section{Proposed Device:}

As a simple illustration, Fig. 1 shows a typical operation related to a coupler in general view. According to this description, one input and at least two outputs are defined for a coupler block. In this regard, the radiation (includes full THz spectrum) is considered as input while transmission part of waves is assumed as regular output. Additionally, reflection channel is considered as coupled output. So seems obvious that absorption must be minimized to obtain high performance operation. In this regard, Fig. 2 reports a unit cell of proposed coupler includes three layers of dielectrics (Sio2, PMMA and SU-8) and four grapheme patterns. The patterns include periodic arrays of graphene disks, ribbons and continuous sheet. 


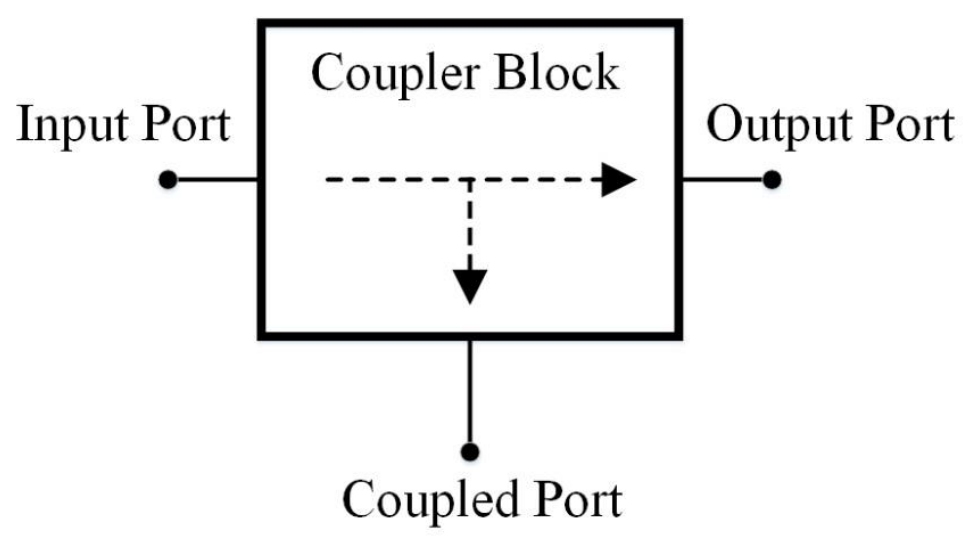

Fig.1. A simple schematic of coupler

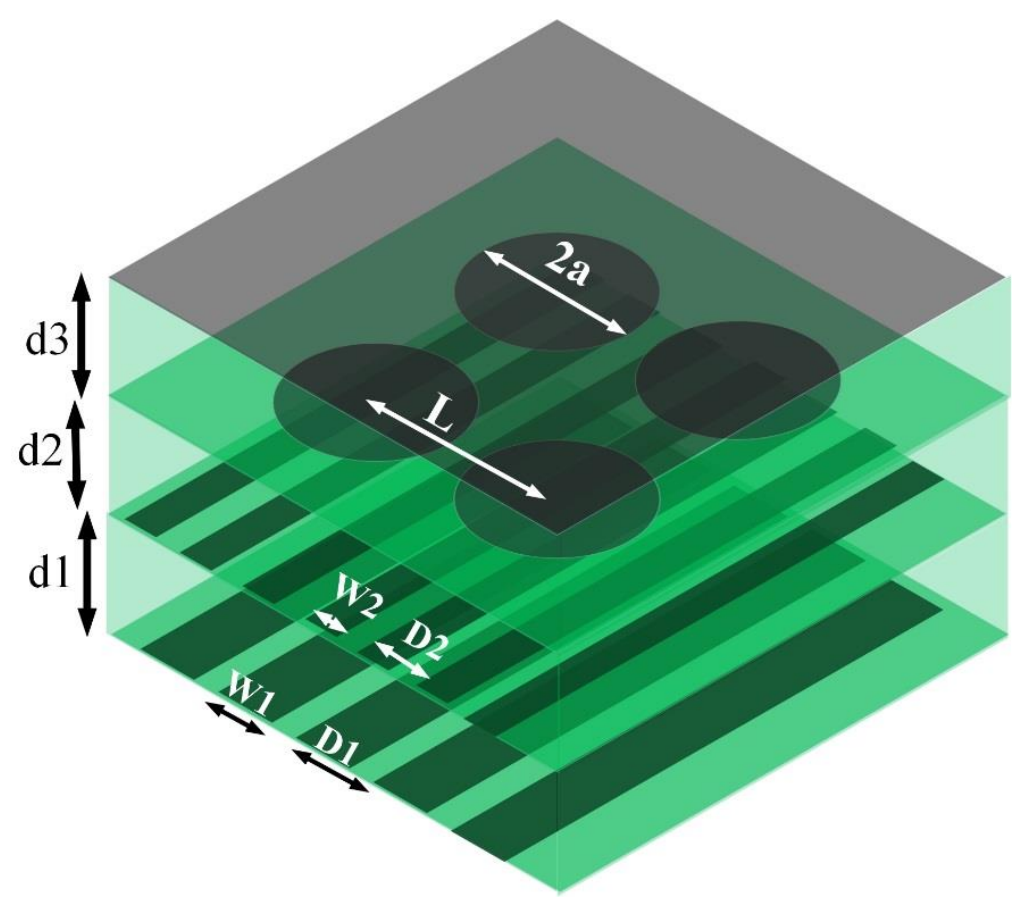

Fig. 2. Proposed structure with specified geometrical aspects.

Dielectric: $d_{1}=12 \mu \mathrm{m}, d_{2}=23 \mu \mathrm{m}, d_{3}=27 \mu \mathrm{m}$

Ribbons: $W_{1}=8.2 \mu \mathrm{m}, D_{1}=4.1 \mu \mathrm{m}, W_{2}=6.5 \mu \mathrm{m}, D_{2}=2.7 \mu \mathrm{m}$

Disks : $2 a=12.4 \mu \mathrm{m}, L=7.1 \mu \mathrm{m}$ 
$d$, a, $\mathrm{L}, \mathrm{W}$, and $\mathrm{D}$ are dielectric thickness, disk radius, disk period, ribbons width, and ribbons period respectively.

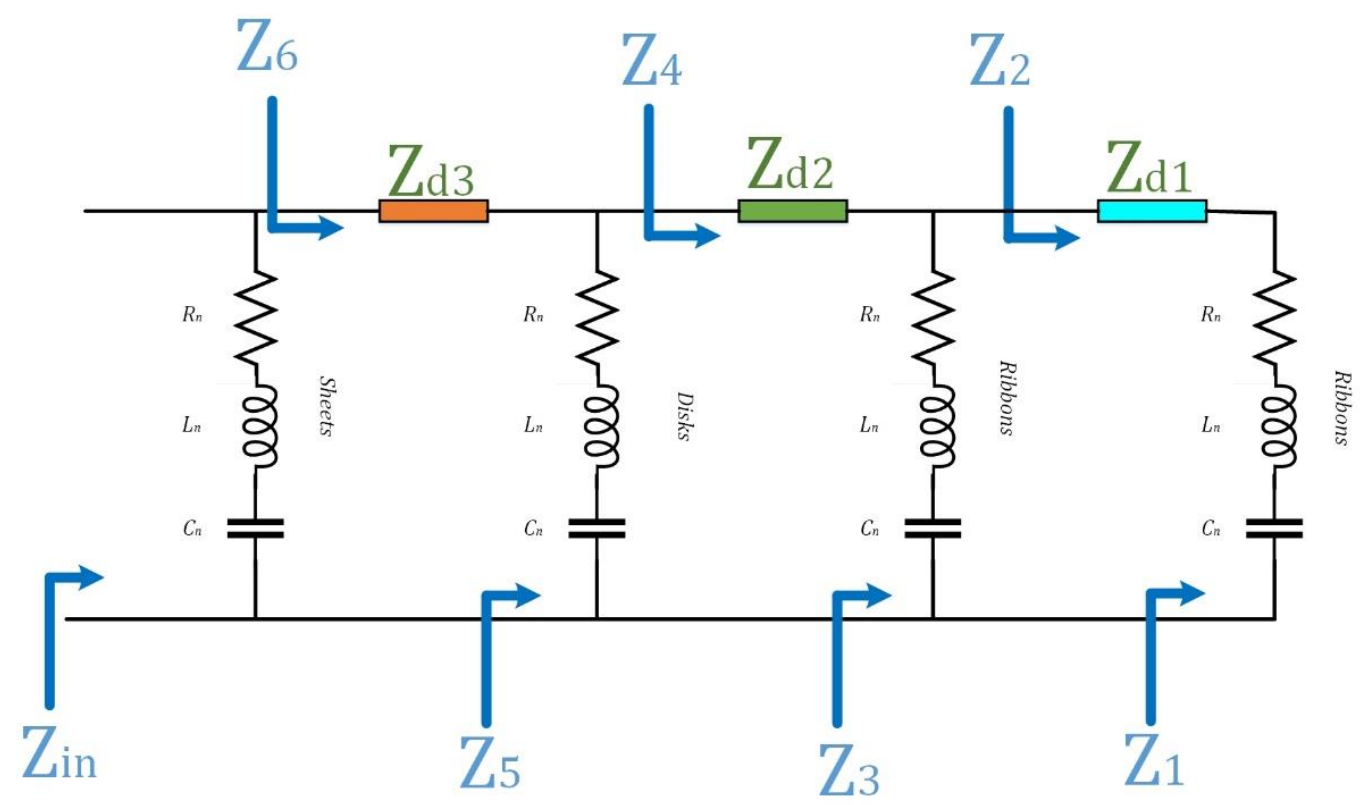

Fig. 3. Equivalent circuit model for the proposed device. The dielectrics 1, 2, and 3 are SU-8, SiO2, and PMMA, So, are modeled as pure imaginary impedance while each graphene pattern is modeled via three parallel RLC branches, each RLC branch is corresponding to one of the existing biases.

In the proposed structure, different dielectrics with different refractive index and of course close to each other are used. The reason for this is to use all the wavelengths of the wave propagated to the device surface. Also, the photoresist dielectric layer such as SU-8, is a promising choice for hybrid metamaterial structures for $\mathrm{THz}$ absorbers and emitters, especially in situations where rapid prototyping, large areas, and simpler means of application (such as painting) are required. Matching simulation to measurements was used to determine the refractive index of SU-8 at THz frequencies, extending the known values from 0.1 to $1.6 \mathrm{THz}$ to as far as $10 \mathrm{THz}$. Here the THz coupler with SU-8 photoresist dielectric layer, assures us that the proposed coupler structure is fully sensitive to the wave. This type of metamaterial, coupled with the applicability of SU-8 as a structural material, offers possibilities for quick, simple microfabrication of THz imagers. SU-8, a negative photoresist, 
is a low-cost material that can quickly be spun onto a substrate at a wide range of thicknesses, and then photolithographically patterned into a variety of structures. It is also transparent to $\mathrm{THz}$ radiation and thus a suitable choice for a dielectric spacer in metamaterials. Other choices for dielectric layer is SiO2 and PMMA dielectrics with refractive index 1.45 and $149 \mathrm{in} \mathrm{THz}$ band [30].

According to [10] and [14], each pattern can be modeled via a resistor, inductor, and capacitor. These elements are related to some physical constants and geometrical sizes as bellow:

Graphene Ribbons :

$$
\begin{aligned}
& R_{n}=\frac{D}{S_{n}^{2}} \frac{\pi \mathrm{h}^{2}}{e^{2} E_{F} \tau} \\
& L_{n}=\frac{D}{S_{n}^{2}} \frac{\pi \mathrm{h}^{2}}{e^{2} E_{F}}=R_{n} \tau \\
& C_{n}=\frac{S_{n}^{2}}{D} \frac{2 \varepsilon_{\text {eff }}}{q_{n}}
\end{aligned}
$$

\section{Graphene Disks :}

$$
\begin{aligned}
R_{n} & =\frac{P^{2} K_{n}}{\pi S_{n}^{2}} \frac{\mathrm{h}^{2}}{e^{2} E_{F} \tau} \\
L_{n} & =\frac{P^{2} K_{n}}{\pi S_{n}^{2}} \frac{\mathrm{h}^{2}}{e^{2} E_{F}}=R_{n} \tau \\
C_{n} & =\frac{\pi^{2} S_{n}^{2}}{P^{2} K_{n}} \frac{\varepsilon_{e f f}}{q_{1 n}}
\end{aligned}
$$

where the definition of parameters of Eq. (1) and Eq. (2) are reported in Table 1:

Table 1. Definition of parameters.

\begin{tabular}{|l|l|l|l|}
\hline parameter & definition & parameter & definition \\
\hline$R_{n}$ & $\begin{array}{l}\text { Equivalent resistance of } \\
\text { series branch }\end{array}$ & $\varepsilon_{\text {eff }}=\frac{\varepsilon_{1}+\varepsilon_{2}}{2}=\frac{n_{1}^{2} \varepsilon_{0}+n_{2}^{2} \varepsilon_{0}}{2}$ & Effective permittivity \\
\hline$L_{n}$ & $\begin{array}{l}\text { Equivalent inductor of } \\
\text { series branch }\end{array}$ & $\varepsilon_{1}$ & $\begin{array}{l}\text { Permittivity of first } \\
\text { substance }\end{array}$ \\
\hline
\end{tabular}




\begin{tabular}{|l|l|l|l|}
\hline$C_{n}$ & $\begin{array}{l}\text { Equivalent capacitor of } \\
\text { series branch }\end{array}$ & $\varepsilon_{2}$ & $\begin{array}{l}\text { Permittivity of second } \\
\text { substance }\end{array}$ \\
\hline$P$ & $\begin{array}{l}\text { Period of graphene } \\
\text { disks }\end{array}$ & $\varepsilon_{0}=8.85 \times 10^{-12} \frac{F}{m}$ & Free space permittivity \\
\hline$D$ & $\begin{array}{l}\text { Period of graphene } \\
\text { ribbons }\end{array}$ & $E_{F}=\frac{\tau e v_{F}^{2}}{\mu}=e \mu_{c}$ & Fermi energy level \\
\hline$K_{n} \cong\left(n-\frac{1}{4}\right) \frac{\pi}{\omega}$ & Eigen value & $\tau=\frac{\mu E_{F}}{e v_{F}^{2}}$ & Electron relaxation time \\
\hline$S_{n}$ & Eigen function & $\mu$ & Electron mobility \\
\hline$q_{n}$ & Eigen value & $\mu_{c}$ & Chemical potential \\
\hline$q_{1 n}$ & Eigen value & $\begin{array}{l}\text { Fermi velocity of } \\
\text { graphene }\end{array}$ & Electron charge \\
\hline$v_{F}=10^{6} \frac{m}{s}$ & \begin{tabular}{l} 
Reduce Plank Constant \\
\hline$\varepsilon_{\text {dielectric }}=n_{\text {dielectric }}^{2} \times \varepsilon_{0}=(1.45)^{2} \times 8.85 \times 10^{-12}=18.6 \times 10^{-12} \frac{F}{m}$
\end{tabular} & $\begin{array}{l}\text { Permittivity of } \\
\text { dielectric (SiO2) }\end{array}$ \\
\hline
\end{tabular}

To obtain $q_{n}$, and $q_{1 n}$, physical parameter such as $a, \mathrm{~L}, W$, and $D$, must be designed and then referred to Table 2 .

Table 2. the value of $q_{n}$ and $q_{1 n}$ based on the geometry of the proposed device.

\begin{tabular}{|l|l|l|l|l|l|l|l|l|l|}
\hline$\frac{2 a}{L}$ & 0.1 & 0.2 & 0.3 & 0.4 & 0.5 & 0.6 & 0.7 & 0.8 & 0.9 \\
\hline Graphene Disks $: a q_{11}$ & 0.539 & 0.536 & 0.533 & 0.530 & 0.527 & 0.499 & 0.472 & 0.444 & 0.417 \\
\hline$\frac{W}{D}$ & 0.1 & 0.2 & 0.3 & 0.4 & 0.5 & 0.6 & 0.7 & 0.8 & 0.9 \\
\hline Graphene Ribbonss $: q_{1} \frac{W}{\pi}$ & 0.734 & 0.725 & 0.710 & 0.689 & 0.658 & 0.620 & 0.571 & 0.507 & 0.420 \\
\hline
\end{tabular}

According to [16] and Fig. 3 as equivalent circuit model, impedance load tends to graphene ribbons $\left(Z_{L}=Z_{\text {graphene-ribbon }}\right)$. So, $Z_{1}$ impedance sees to graphene ribbons at the end of the line. Therefore, $Z_{1}$ calculated by Eq. (3). Then Eq. (4) is obtained based on the transmision line 
concept [16]. Also, the dielectric can be modeled via Eq. (15). And the input impedance is calculated as below:

$$
\begin{aligned}
& Z_{1}=Z_{\text {graphene-ribbon }} \\
& Z_{2}=Z_{d_{1}} \frac{Z_{1}+j Z_{d_{1}} \times \tan \left(\beta_{d} d_{1}\right)}{Z_{d_{1}}+j Z_{1} \cdot \tan \left(\beta_{d} d_{1}\right)} \\
& Z_{d_{1}}=\frac{Z_{0}}{n_{S U-8}} \\
& Z_{3}=Z_{2} \| Z_{\text {graphene-ribbon }}=\frac{Z_{2} \times Z_{\text {graphene-ribbon }}}{Z_{2}+Z_{\text {graphene-ribbon }}} \\
& Z_{4}=Z_{d_{2}} \frac{Z_{3}+j Z_{d_{2}} \cdot \tan \left(\beta_{d} d_{2}\right)}{Z_{d_{2}}+j Z_{3} \cdot \tan \left(\beta_{d} d_{2}\right)} \\
& Z_{d_{2}}=\frac{Z_{0}}{n_{\text {Sio }}} \\
& Z_{5}=Z_{4} \| Z_{\text {graphene-disks }}=\frac{Z_{4} \times Z_{\text {graphene-disks }}}{Z_{4}+Z_{\text {graphene-disks }}} \\
& Z_{6}=Z_{d_{3}} \frac{Z_{5}+j Z_{d_{3}} \cdot \tan \left(\beta_{d} d_{3}\right)}{Z_{d_{3}}+j Z_{5} \cdot \tan \left(\beta_{d} d_{3}\right)} \\
& Z_{d_{3}}=\frac{Z_{0}}{n_{P M M A}} \\
& Z_{\text {in }}=Z_{6} \| Z_{\text {graphene-sheet }}=\frac{Z_{6} \times Z_{\text {graphene-sheet }}}{Z_{6}+Z_{\text {graphene-sheet }}}
\end{aligned}
$$

where the definition of parameters of Eq. (13) to Eq. (22) are reported in Table 3:

Table 3. Definition of parameters.

\begin{tabular}{|l|l|}
\hline Parameter & Definition \\
\hline$\beta_{d}$ & The wave propagation constant in the dielectric substrate. \\
\hline$d$ & The dielectric thickness. \\
\hline$Z_{0}$ & The free space impedance and equal to $120 \pi \Omega$. \\
\hline$n_{d}$ & Refractive index of dielectric. \\
\hline
\end{tabular}


Since the main goal in designing the proposed device is to achieve the maximum transmission and/or reflection against bias changing, calculating device impedance is inevitable to verify the matching concept and knowing absorption, transmission, and reflection percentage in the desired frequency range. In another word, if $Z_{\text {in }} \approx Z_{0} \approx 120 \pi$, the device can achieve maximum absorption while far values of input impedance compared to $120 \pi$, minimized absorption and increase total transmission and reflection.

\section{Simulation Results:}

According to Eq. (13), changing gate bias leads to changing chemical potential which forces the device to react differently [17].

$$
V_{g}=\frac{\mu_{c}^{2} e d}{\mathrm{~h}^{2} v_{f}^{2} \varepsilon_{0} \varepsilon_{\text {dielectric }} \pi}
$$

Where $V_{g}, \varepsilon_{r}, \varepsilon_{0}, d$, and $v_{f}$ are respectively the gate voltage, the permittivity of the dielectric substrate, the permittivity of free space, the thickness of the dielectric substrate, and the Fermi velocity. The values of the parameters of Eq. (23) are given in Table 1.

In this regard, full wave simulation based on Finite Element Method (FEM) is exploited to show device performance. Fig. 5 extracted from CST simulator which shows transmission, reflection and absorption simultaneously. 


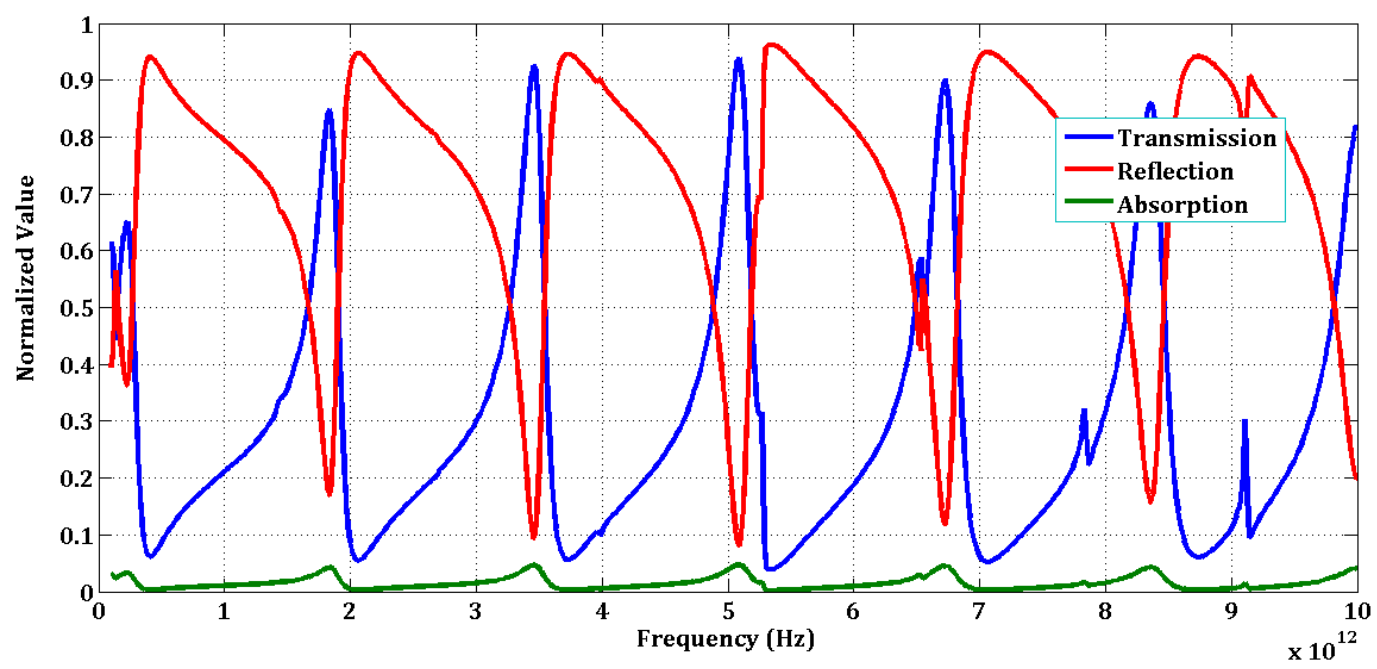

Fig. 4. Transmission, Reflection and Absorption of the proposed structure.

According to Fig. 4, the proposed device shows multi-band operation for both transmission and reflection. To investigate device sensitivity against design parameters, ample simulations are performed. In this regard, Fig. 5, Fig. 6 and Fig. 7 are expressed to show both transmission and reflection variations versus layers thicknesses.
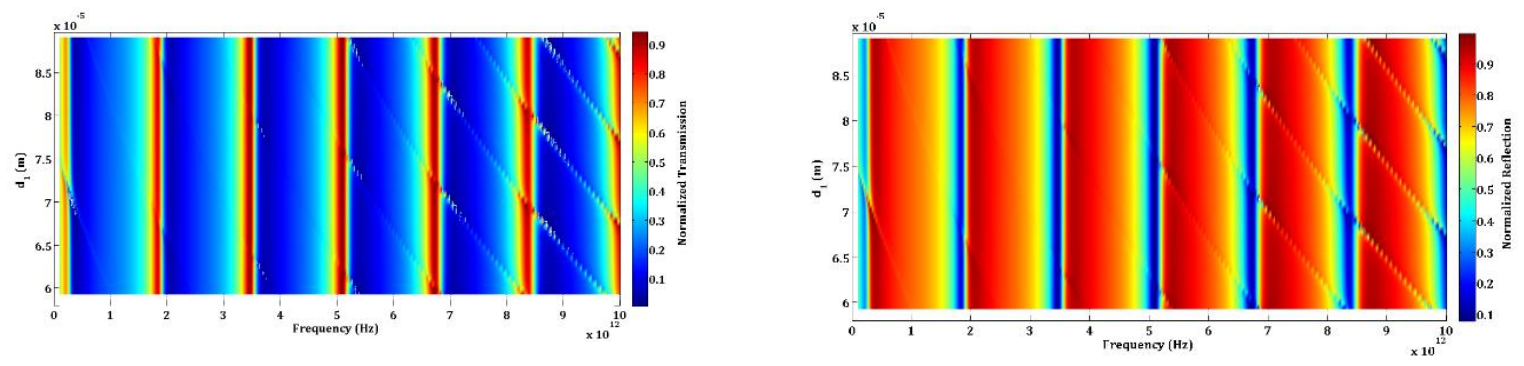

Fig. 5. Variations of transmission (Left) and reflection (Right) versus first layer $\left(d_{1}\right)$ thickness.
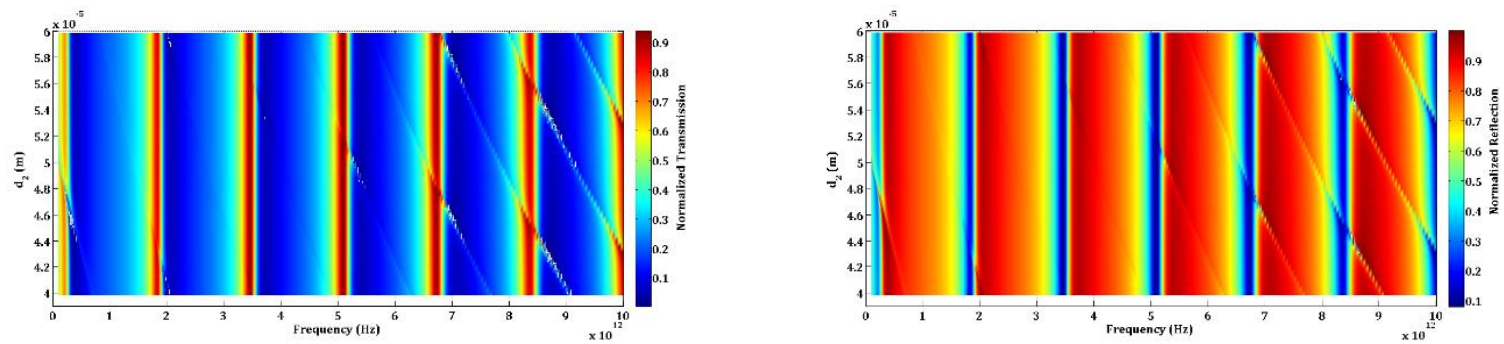

Fig. 6. Variations of transmission (Left) and reflection (Right) versus second layer $\left(\mathrm{d}_{2}\right)$ thickness. 

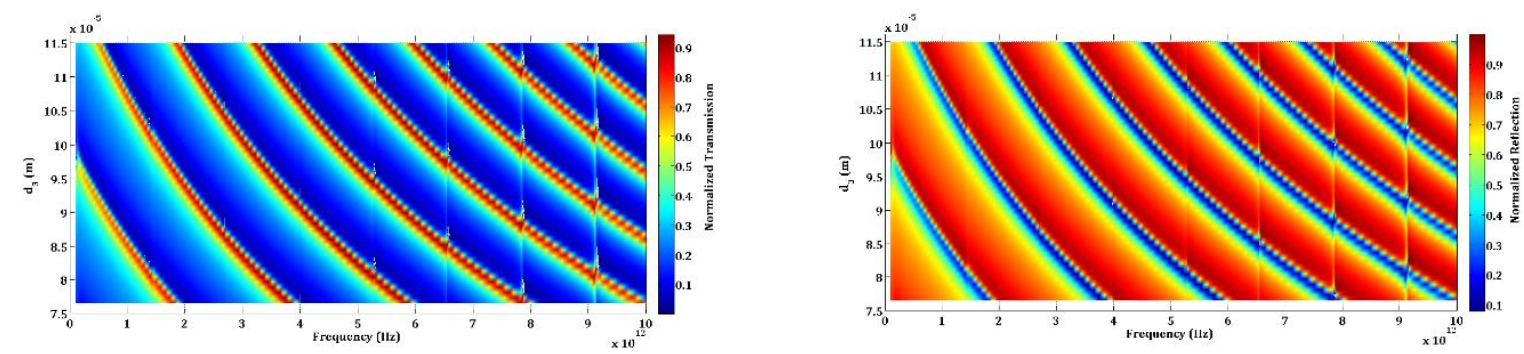

Fig. 7. Variations of transmission (Left) and reflection (Right) versus third layer $\left(d_{3}\right)$ thickness.

According to these Fig. 5, Fig. 6, and Fig. 7, variations of the third layer shifts peaks of transmission and reflection considerably while operation is relatively fixed against variations of first- and second-layers thicknesses. This stems from the fact that the third layer thickness is larger than two other layers. Additionally, device performance against chemical potentials variations are reported via Fig. 8, Fig. 9, Fig. 10 and Fig. 11.
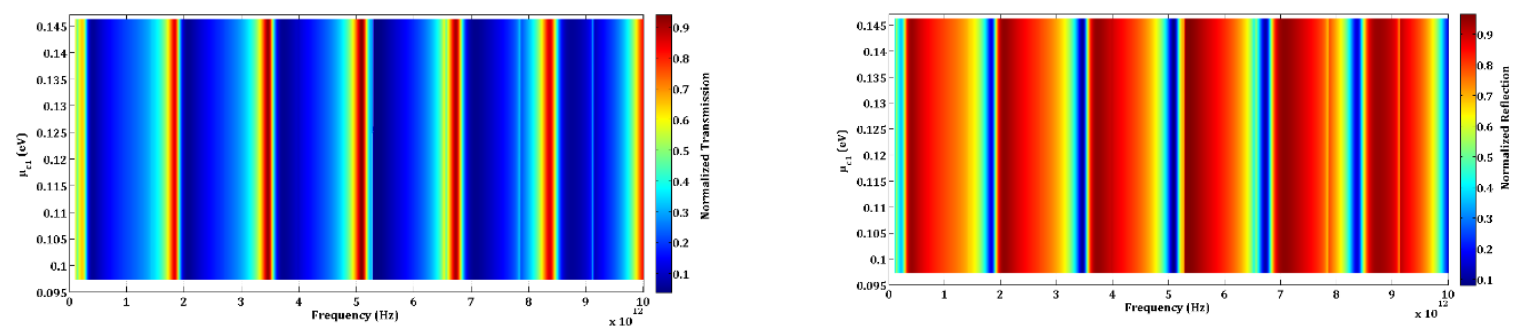

Fig. 8. Variations of transmission (Left) and reflection (Right) versus $\mu_{\mathrm{c} 1}$.
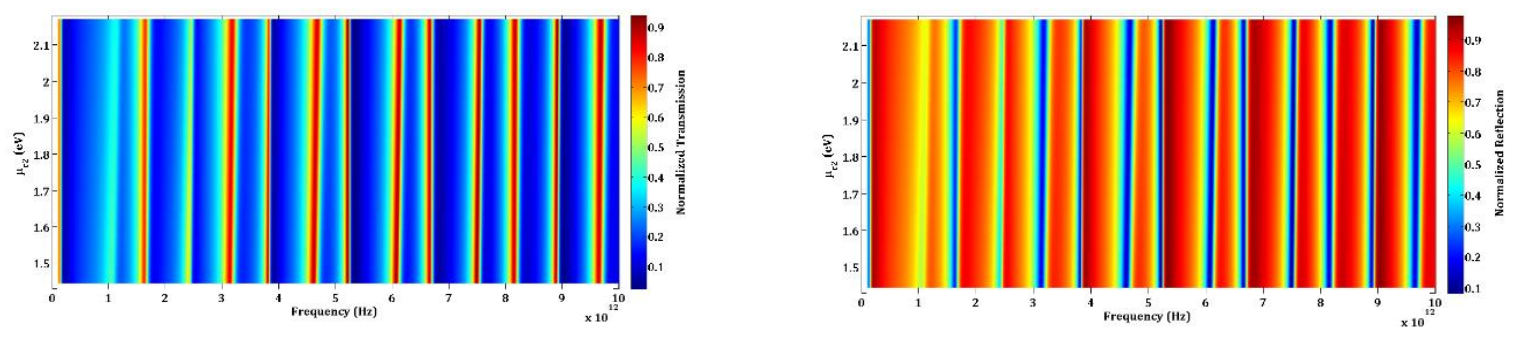

Fig. 9. Variations of transmission (Left) and reflection (Right) versus $\mu_{\mathrm{c} 2}$. 

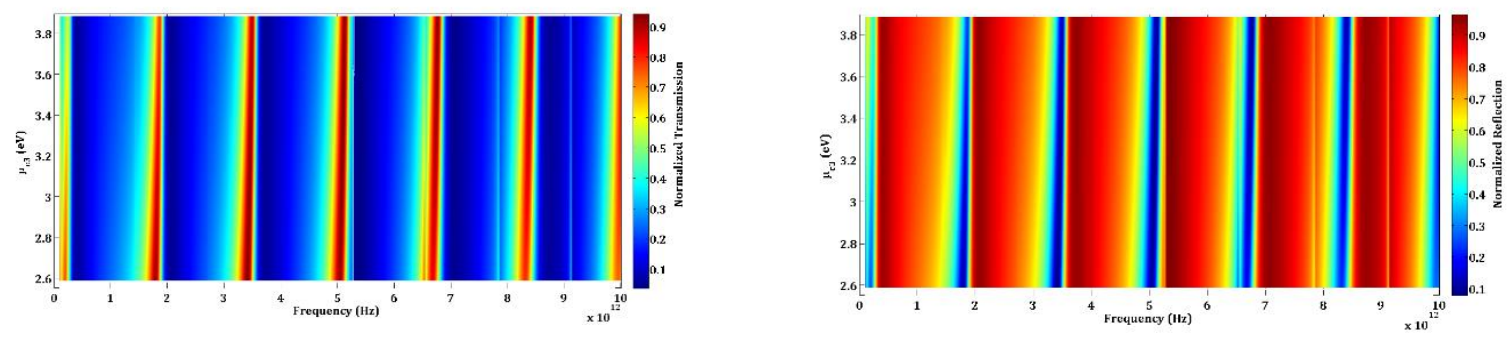

Fig. 10. Variations of transmission (Left) and reflection (Right) versus $\mu_{\mathrm{c} 3}$.
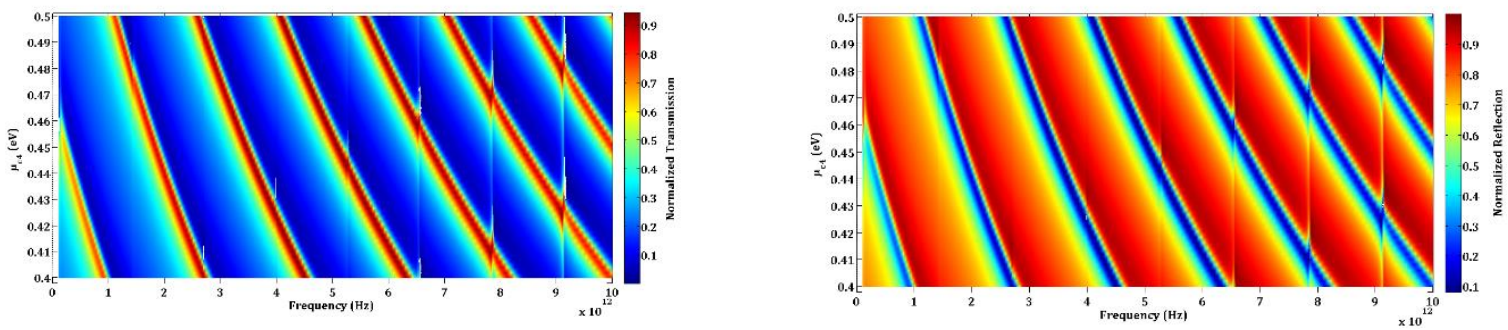

Fig. 11. Variations of transmission (Left) and reflection (Right) versus $\mu_{\mathrm{c} 4}$.

At last but not least, transmission and reflection variations versus changing electron relaxation time are reported by Fig. 12 .
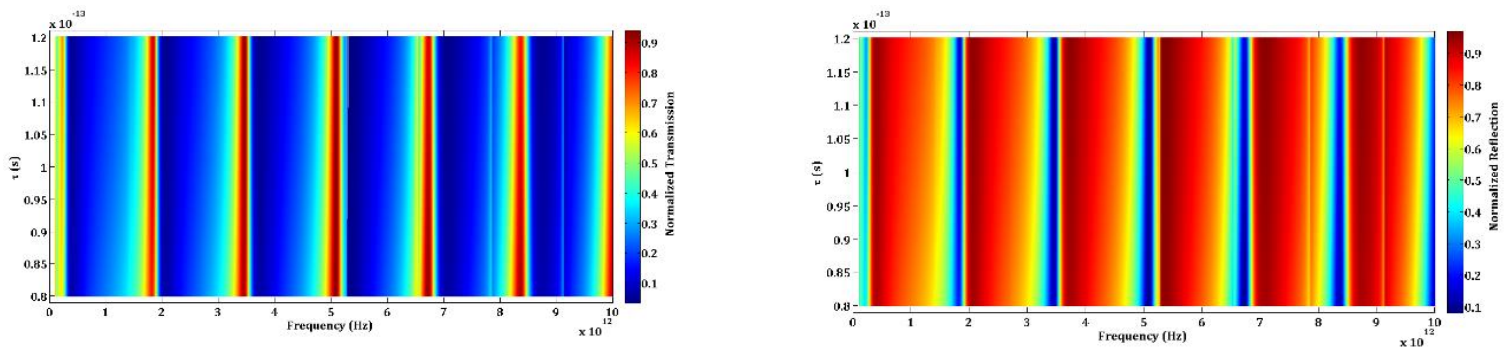

Fig.12. Variations of transmission (Left) and reflection (Right) versus electron relaxation time. 
Finally, the comparison table is reported in Table 5.

Table 5. Comparison table.

\begin{tabular}{|c|c|c|c|c|c|c|}
\hline Ref & $\begin{array}{c}\text { Central Frequency } \\
(\mathrm{THz})\end{array}$ & $\begin{array}{c}\text { Absorption } \\
(\%)\end{array}$ & $\begin{array}{c}\text { Transmission } \\
(\%)\end{array}$ & $\begin{array}{c}\text { Reflection } \\
(\%)\end{array}$ & $\begin{array}{c}\text { Total } \\
\text { Height } \\
(\mu m)\end{array}$ & Structure \\
\hline$[24]$ & $0.94,2.69$ & $>95,>83$ & 0 & $<5,<7$ & 95.2 & $\begin{array}{c}\text { Dielectric1 pattern/non- } \\
\text { structured } \\
\text { graphene/dielectric1/metal }\end{array}$ \\
\hline$[25]$ & $1.8,2.4$ & $>95,>95$ & 0 & $<5,<5$ & 4.5 & $\begin{array}{c}\text { square metallic patches/ ultra- } \\
\text { thin insulator dielectric layer/ } \\
\text { continuous metallic ground }\end{array}$ \\
\hline$[26]$ & $3.6,4.7$ & $>90,>85$ & 0 & $<10,<15$ & 120 & $\begin{array}{c}\text { SiO2/Graphene/Polyimide/Si/ } \\
\text { Al2O3/Substrate }\end{array}$ \\
\hline$[27]$ & 188,225 & $>95,>95$ & 0 & $<5,<5$ & 0.77 & $\begin{array}{c}\text { Polymethyl Methacrylate } \\
\text { (PMMA)/Graphene/SiO2/Au }\end{array}$ \\
\hline$[28]$ & 6,25 & $>97,>95$ & 0 & $<3,<5$ & 25 & $\begin{array}{c}\text { Graphene/Polysilicon/Dielectr } \\
\text { ic Slab/Perfect Conductor }\end{array}$ \\
\hline$[29]$ & 3,8 & $>99,>99$ & 0 & $<1,<1$ & 40 & $\begin{array}{c}\text { Multi Bias Graphene Ribbons } \\
\text { twice)/TOPAS (twice)/Au }\end{array}$ \\
\hline This & $1.98,3.49,5.1,6.7$, & $<.3$ & $<5$ & $<10$ & $\begin{array}{c}\text { Graphene Ribbons/ SU-8/ } \\
\text { Graphene Ribbons/ SiO2/ } \\
\text { Graphene Disks/ PMMA/ } \\
\text { Graphene Sheets }\end{array}$ \\
\hline
\end{tabular}

\section{Conclusion:}

Using equivalent circuit model and full wave simulations, a multi-layer structure based on graphene patterns is presented. The reflection and transmission channels are considered as outputs while aim is minimizing absorption. Simulations results show both reflection and transmission in entire $\mathrm{THz}$ spectrum while ample sensitivity analysis are reported versus geometrical parameters and gate biasing. According to the results, the proposed structure can be considered as an appropriate coupler block in THz spectrum. 


\section{References:}

[1] A. Y. Pawar, D. D. Sonawane, K. B. Erande, and D. V. Derle, "Terahertz technology and its applications," Drug Invention Today, vol. 5, no. 2, pp. 157-163, 2013.

[2] S. Yamaguchi, Y. Fukushi, O. Kubota, T. Itsuji, T. Ouchi, and S. Yamamoto, "Brain tumor imaging of rat fresh tissue using terahertz spectroscopy," Scientific Reports, vol. 6, p. 30124, 2016.

[3] Yoon, Junyoung, et al. "Development of the antenna adjustable power coupler for 325 MHz superconducting cavities." Nuclear Instruments and Methods in Physics Research Section A: Accelerators, Spectrometers, Detectors and Associated Equipment 1010 (2021): 165484.

[4] I. F. Akyildiz, J. M. Jornet, and C. Han, "Terahertz band: Next frontier for wireless communications," Physical Communication, vol. 12, pp. 16-32, 2014.

[5] Tang, Y., C-F. Wu, and L. Wang. "Thermal analysis for fundamental power coupler of HALF 499.8 MHz superconducting cavity." Journal of Instrumentation 16.06 (2021): T06010.

[6] S. Koenig, D. Lopez-Diaz, J. Antes, F. Boes, R. Henneberger, A. Leuther, A. Tessmann, R. Schmogrow, D. Hillerkuss, R. Palmer et al., "Wireless sub-THz communication system with high data rate," Nature Photonics, vol. 7, no. 12, p. 977, 2013.

[7] R. N. Mitra and D. P. Agrawal, “5G mobile technology: A survey,” ICT Express, vol. 1, no. 3, pp. 132-137, 2015.

[8] Sangroula, M. P., D. Winklehner, and M. Schuett. "Design and optimization of the RF inputcoupler for a low-frequency, split-coaxial RFQ." Journal of Instrumentation 16.08 (2021): P08061.

[9] Geim, Andre K., and Konstantin S. Novoselov. "The rise of graphene." Nanoscience and technology: a collection of reviews from nature journals. 2010. 11-19.

[10] Khavasi, Amin, and Behzad Rejaei. "Analytical modeling of graphene ribbons as optical circuit elements." IEEE Journal of Quantum Electronics, Vol. 50, no. 6, PP. 397-403, 2014.

[11] Barzegar-Parizi, Saeedeh, Behzad Rejaei, and Amin Khavasi. "Analytical circuit model for periodic arrays of graphene disks." IEEE Journal of Quantum Electronics, Vol. 51, no. 9, PP. 1-7, 2015. 
[12] Psychogiou, Dimitra, and Roberto Gómez-García. "Tunable reflectionless microstrip bandpass filters." 2018 IEEE Radio and Wireless Symposium (RWS). IEEE, 2018.

[13] Gómez-García, Roberto, et al. "Wide-band signal-interference duplexer with contiguous single/dual-band channels and its application to quasi-absorptive bandpass filters." Electronics Letters 54.9 (2018): 578-580.

[14] Aghaee, Toktam, and Ali A. Orouji. "Reconfigurable multi-band, graphene-based THz absorber: Circuit model approach." Results in Physics 16 (2020): 102855.

[15] Soltani-Zanjani, Masoud, et al. "Multi-bias graphene-based THz super absorber." Results in Physics 25 (2021): 104326.

[16] Cheng, David Keun. "Field and wave electromagnetics". Pearson Education India, 1989.

[17] Hlali, Aymen, Zied Houaneb, and Hassen Zairi. "Dual-band reconfigurable graphenebased patch antenna in terahertz band: Design, analysis and modeling using WCIP method." Progress In Electromagnetics Research 87 (2018): 213-226.

[18] Luo, Yanbin, et al. "Graphene-based multi-beam reconfigurable THz antennas." IEEE Access 7 (2019): 30802-30808.

[19] Patel, Shobhit K., et al. "Design of graphene metasurface based sensitive infrared biosensor." Sensors and Actuators A: Physical 301 (2020): 111767.

[20] Charola, Shreyas, et al. "Broadband graphene-based metasurface solar absorber." Microwave and Optical Technology Letters 62.3 (2020): 1366-1373.

[21] Huang, Yi, et al. "Design of a beam reconfigurable THz antenna with graphene-based switchable high-impedance surface." IEEE Transactions on Nanotechnology 11.4 (2012): 836-842.

[22] Patel, Shobhit K., et al. "Sensitivity analysis of metasurface array-based refractive index biosensors." IEEE Sensors Journal 21.2 (2020): 1470-1477.

[23] Parmar, Juveriya, and Shobhit K. Patel. "Tunable and highly sensitive graphene-based biosensor with circle/split ring resonator metasurface for sensing hemoglobin/urine biomolecules." Physica B: Condensed Matter (2021): 413399.

[24] Qi, Limei, Chang Liu, and Syed Mohsin Ali Shah. "A broad dual-band switchable graphene-based terahertz metamaterial absorber." Carbon 153 (2019): 179-188. 
[25] Wang, Ben-Xin, et al. "Design of a dual-band terahertz metamaterial absorber using two identical square patches for sensing application." Nanoscale Advances 2.2 (2020): 763769.

[26] Cai, Yijun, et al. "Tunable dual-band terahertz absorber with all-dielectric configuration based on graphene." Optics Express 28.21 (2020): 31524-31534.

[27] Wu, Pinghui, et al. "A narrow dual-band monolayer unpatterned graphene-based perfect absorber with critical coupling in the near infrared." Micromachines 11.1 (2020): 58.

[28] Barzegar-Parizi, Saeedeh, and Amin Khavasi. "Designing dual-band absorbers by graphene/metallic metasurfaces." IEEE Journal of Quantum Electronics 55.2 (2019): 1-8.

[29] Aghaee, Toktam, and Ali A. Orouji. "Manipulating pattern periods via external bias for graphene-based THz Dual-Band absorber." Microwave and Optical Technology Letters (2021).

[30] Grbovic, Dragoslav, et al. "Metal-organic hybrid resonant terahertz absorbers with SU8 photoresist dielectric layer." Journal of Micro/Nanolithography, MEMS, and MOEMS 12.4 (2013): 041204. 